\title{
Microbial Keratitis after Penetrating Keratoplasty
}

\author{
A. K. BATES, C. M. KIRKNESS, L. A. FICKER, A. D. McG. STEELE and N. S. C. RICE \\ London
}

\begin{abstract}
Summary
Thirty cases of microbial keratitis after penetrating keratoplasty were reviewed to examine the associated risk factors, the spectrum of pathogens and the prognosis for graft survival and visual outcome.

The indications for keratoplasty in this group differed markedly from those for all corneal grafts performed with a much higher incidence of previous microbial keratitis and of herpes simplex keratitis.

A positive culture was obtained in $93 \%$ of cases and in contrast to microbial keratitis overall, Gram positive organisms predominated particularly streptococcus pneumoniae and staphlycoccus aureus.

Risk factors identified were loose or broken sutures, graft decompensation and a poor ocular surface environment.

There was a poor prognosis for graft survival with only $23 \%$ of cases retaining a clear graft. Overall $53 \%$ of cases were regrafted.
\end{abstract}

Microbial keratitis is an uncommon complication of penetrating keratoplasty. Reported rates of incidence are low, ranging from $1.9-4.9 \% .^{1,2}$ The ocular morbidity is serious with a large proportion of affected patients losing graft clarity and requiring further surgical intervention.

This study of microbial keratitis following penetrating keratoplasty was undertaken to examine the spectrum of pathogens, to identify the associated risk factors and to evaluate the prognosis for graft survival and visual outcome.

\section{Patients and Methods}

Case-notes of patients under the care of the Corneal Clinic, Moorfields Eye Hospital between 1983 and 1988 who had undergone penetrating keratoplasty and subsequently developed microbial keratitis, confirmed by Gram stain or histopathology and, or culture from scrapes or corneal biopsy, were reviewed.

The following information was recorded for each case: age, sex, indication for penetrating keratoplasty, time between penetrating keratoplasty and microbial keratitis, site of keratitis, bacteriological findings, potential risk factors for keratitis and outcome in terms of visual acuity and fate of the graft.

A positive culture was defined as growth of more than one colony of an organism in the inoculating streak of any culture medium.

In most cases multiple colonies were grown. Culture media routinely used were blood agar, Sabouraud's medium, thioglycollate medium, nutrient broths and where indicated Eschrichia Coli seeded non-nutrient agar for Acanthamoeba.

From the Corneal Clinic and Pocklington Eye Transplantation Unit, Department of Clinical Ophthalmology, Institute of Ophthalmology, Moorfields Eye Hospital.

Correspondence to: A. K. Bates FRCS, Moorfields Eye Hospital, City Road, London EC1V 2PD. 
Table I Indications for penetrating keratoplasty for the grafts in this series

\begin{tabular}{llc}
\hline & $\begin{array}{c}\text { No. of } \\
\text { cases }\end{array}$ & $\begin{array}{c}\% \text { of } \\
\text { cases }\end{array}$ \\
\hline Microbial keratitis in previous graft & 8 & 27 \\
Herpes simplex keratitis & 7 & 23 \\
Aphakic bullous keratopathy & 5 & 17 \\
Interstitial keratitis & 2 & 7 \\
Cicatrising conjunctival disease & 2 & 7 \\
Other indications & 6 & 20 \\
\hline
\end{tabular}

\section{Results}

Thirty cases of microbial keratitis were identified in 22 patients after penetrating keratoplasty. Approximately 1700 keratoplasties were performed over this period suggesting an incidence of $1.76 \%$. Seven patients had two episodes of microbial keratitis in different grafts involving different organisms. One patient had two episodes of infection in the same graft which were separated by six months and also involved different organisms.

The mean age at presentation with microbial keratitis was 55 years (range 20-80) and $59 \%$ were male. The median interval between penetrating keratoplasty and microbial keratitis was ten months (range 1-168). Eleven $(37 \%)$ infections occurred within the first six months after surgery. In patients for whom the indication for penetrating keratoplasty had been microbial keratitis, the median interval between penetrating keratoplasty and microbial keratitis was three months (range 1-10). There were eight such cases.

The indication for penetrating keratoplasty in this group of patients is shown in Table $I$. The largest group consisted of eight patients who had microbial keratitis in a previous graft which had resulted in perforation or had not adequately responded to medical treatment. In the seven patients with herpes simplex keratitis, four were grafted for incipient or actual corneal perforation, two for central scarring and one for corneal decompensation secondary to herpetic uveitis. Other indications included rheumatoid melt, keratoconus, perforation of a congenital leukoma, Acanthamoeba keratitis and corneal decompensation after microbial keratitis and multiple surgery for congenital glaucoma.

In total, $47 \%$ of the grafts performed in this group of patients were tectonic for actual or incipient corneal perforation and, in 30\% there was corneal decompensation at the time of keratoplasty.

Microbial keratitis occurred in 15 cases wholly within the graft and in 15 cases at the graft-host interface. In the latter group ten had either a broken or loose suture at the site of the infection, or a suture had been removed within the previous four weeks.

Of the 30 infections, a positive microbiological diagnosis was made on the basis of a corneal scrape and subsequent positive culture in 28 , confirmed in one case by an anterior chamber paracentesis. In one case, Streptococcus pneumoniae was cultured from a corneal button, following repeat keratoplasty, after negative corneal scrapes. In one case, the bacteriological diagnosis was equivocal after corneal scrapes; Gram positive cocci were seen on the Gram stain which were thought to be Streptococcus but the organisms could not be cultured.

The organisms isolated are shown in Table II. Bacterial infection was implicated in 25 cases and fungal infection in four cases. There was mixed infection in only one case, where Candida albicans and Acanthamoeba were isolated. Gram positive organisms were involved in 19 cases $(68 \%)$, predominantly Stretococcus pneumoniae and Staphylococcus aureus. Gram negative organisms were cultured from only six cases $(20 \%)$, Moraxella spp in four and Pseudomonas aeruginosa in two cases. In five cases the microbial keratitis had crystalline morphology, three occurred within the graft and two at the graft-host interface. Streptococcus pneumoniae was isolated from two cases, Streptococcus viridans from two cases, and one case had equivocal culture

Table II Organisms isolated

No. of cases

\begin{tabular}{ll}
\hline Streptococcus pneumoniae & 8 \\
Staphylococus aureus & 6 \\
Candida albicans & 4 \\
Moraxella spp & 4 \\
Staphylococcus epidermidis & 2 \\
Streptococcus viridans & 2 \\
Pseudomonas aeruginosa & 2 \\
Anaerobic streptococcus & 1 \\
Acanthamoeba & 1
\end{tabular}


Table III Risk factors

No. of cases

\begin{tabular}{lr}
\hline Graft & 10 \\
Suture related problems & 4 \\
Decompensated graft & 3 \\
Recent rejection episode & 3 \\
Epithelial defect & 1 \\
Contact lens in situ & 1 \\
Wound dehiscence & \\
Ocular surface & 7 \\
$\quad$ Mucosal & 7 \\
Dry eye & 4 \\
Trichiasis & \\
Systemic & \\
Atopy & 6 \\
\hline
\end{tabular}

but was thought to be a Streptococcus spp on the basis of Gram stain.

A number of potential risk factors for microbial keratitis were examined, including the medications being used at the onset of infection. Twenty nine cases were receiving topical steroid therapy, 16 of whom were taking a dose of $\mathrm{G}$. Prednisolone $0.3 \%$ four times a day or greater. Only 12 cases were receiving prophylactic antibiotic therapy; in eight, this consisted of $\mathrm{G}$. chloramphenicol $0.5 \%$ four times a day. In six of the 12 cases, the organism cultured was sensitive in vitro to the prophylactic antibiotic currently in use.
The organisms cultured from these 12 patients were $S$. pneumoniae in four cases, $S$. aureus in three cases, Candida albicans in two cases and Moraxella, Pseudomonas and Acanthamoeba in one case. Four cases were receiving antiviral therapy and 10 cases treatment for glaucoma.

A number of local risk factors were identified. (Table III and Figure 1) Ten (33\%) of the cases were associated with a poor ocular environment. Seven had keratoconjunctivitis sicca, seven mucosal scarring and four trichiasis. One third of patients had a suture related problem, either a broken or loose suture or a suture which had been removed in the previous four weeks. A further third had a compromised graft which was decompensated, had recently suffered a rejection episode or had an epithelial defect. The only systemic risk factor which could be identified was atopy, present in six cases. None of the patients was diabetic. Some patients showed more than one risk factor which is demonstrated in Figure 1.

The outcome of the episode of microbial keratitis is shown in Table IV. Of the patients who retained a clear graft, best corrected visual acuity was $6 / 18$ or better in all but one case. All the patients with perforated corneas underwent repeat penetrating keratoplasty. Of the four cases which developed endoph-

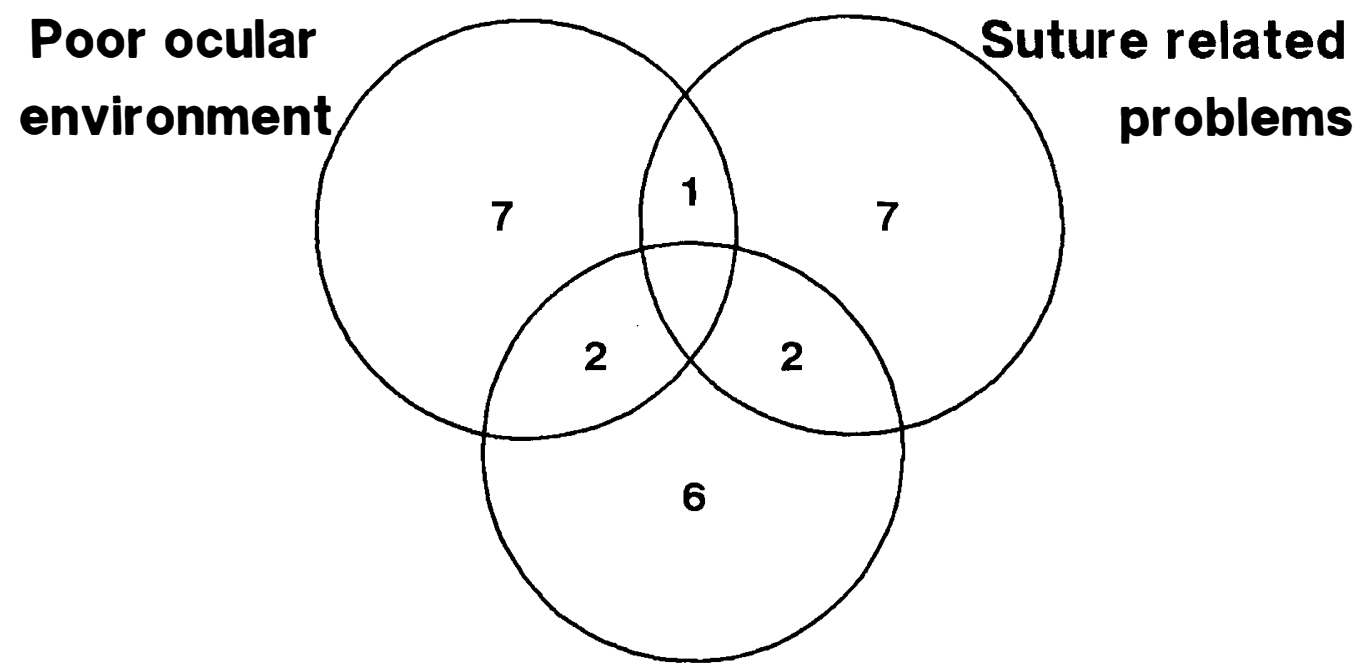

\section{Compromised graft}

Fig. 1. A Venn diagram showing how risk factors overlapped in several instances. 
Table IV Outcome

\begin{tabular}{lcc}
\hline & No. of cases & $\%$ of cases \\
\hline Clear graft & 7 & 23 \\
Decompensated graft & 4 & 13 \\
Corneal perforation & 5 & 17 \\
Endophthalmitis & 4 & 13 \\
Regrafted & 16 & 53 \\
\hline
\end{tabular}

thalmitis, three were eviscerated and one was regrafted.

\section{Discussion}

This study demonstrates that microbial keratitis is a serious, if infrequent, complication following penetrating keratoplasty with high risk of loss of graft clarity.

The majority of cases occur within the first year after surgery $(63 \%)$ particularly in those patients with a recent history of microbial keratitis. Infection may occur much later in association with longer term risk factors such as graft decompensation.

The indications for penetrating keratoplasty in this group of patients differ quite markedly from the overall indications within this institution during the same period of time shown in Table $\mathrm{V}^{3}$ Keratoplasty for herpes simplex keratitis for example constituted only $3 \%$ of all grafts performed whereas in this series of patients, $23 \%$ of grafts were associated with this diagnosis. Clearly certain conditions carry a much higher risk of developing microbial keratitis than others and, in most series, herpetic infection appears to carry a high risk, perhaps because recurrent herpetic disease disrupts the epithelial barrier to infection. Keratoplasty in eyes with a history of microbial keratitis, particularly, if this has not been controlled medically or has resulted in perforation is another factor. The number of grafts performed in this series for tectonic causes was $43 \%$ compared to $6 \%$ of grafts overall. Such cases would seem to have high risk of infection.

There was a much higher rate of positive culture $(93 \%)$ in this series than in previous studies of microbial keratitis, ${ }^{4}$ perhaps because of the severity of infection in many cases, but also because of the diligence with which infecting agents were sought. As in other series of microbial keratitis following penetrating keratoplasty Gram positive organisms predominated (68\%) with Streptococcus pneumoniae and Staphylococcus aureus most commonly isolated..$^{5-7}$ This differs from findings for all cases of microbial keratitis where Gram negative organisms are relatively more important, particularly Pseudomonas aeruginosa, and Streptococcus pneumoniae is less common. ${ }^{4}$

In several infections, there was a crystalline morphology and in common with some reports, these were all Streptococcal in origin. ${ }^{8}$ Interestingly, however, more occurred within the graft than at the graft host interface failing to support the hypothesis that the cytological architecture of the interface is implicated in the creation of the crystalline appearance. ${ }^{9}$

Several studies have suggested that prolonged prophylactic antibiotic therapy combined with steroid therapy after keratoplasty may constitute an iatrogenic risk factor for microbial keratitis. ${ }^{1,5,6,10}$ In this series almost all patients were receiving steroid therapy and $40 \%$ were receiving antibiotics. The spectrum of organisms cultured in patients receiving such antibiotics, however, did not appear to differ significantly from the series as a whole although numbers were too small to allow statistical analysis.

Loose sutures which breach the epithelial surface may provide a direct route for infection into the stroma. Furthermore, the mucus and other foreign material which tends to accumulate around them may become colonised by pathogens. This together with the

Table V Indications for keratoplasty 1985-87

\begin{tabular}{lc}
\hline & \% of cases \\
\hline Keratoconus & 32 \\
Regrafts & 17 \\
Bullous keratopathy & 13 \\
Corneal dystrophies & 9 \\
Fuchs' dystrophy & 6 \\
Interstitial keratitis & 4 \\
Viral keratitis & 3 \\
Suppurative keratitis & 3 \\
Corneal degeneration & 2 \\
Other indications & 2 \\
Trauma & 2 \\
Corneal scarring & 2 \\
Limbal lesions & 1 \\
Melting disorders & 1 \\
\hline
\end{tabular}


associated loss of epithelial integrity makes infection highly likely. The most important risk factor identified in this as in many other series was a suture related problem which occurred in a third of cases. ${ }^{5,6}$ This suggests that patients should be followed closely and should sutures loosen or break they should be removed forthwith. This demonstrates one of the dilemmas that corneal surgeons encounter. To avoid a suture related problem, it would be advisable to remove electively corneal sutures once the wound was secure, but in doing so, the surgeon risks producing astigmatism or even wound dehiscence or rupture. The latter complication may occur despite the most careful clinical assessment. The surgeon, nevertheless, should be aware of the risks of leaving sutures in situ for long enough for biodegradation to occur.

Graft decompensation may result in frank epithelial oedema which allows a breach in the normal epithelial barrier to infection. The ocular environment is of great importance to survival of a corneal graft and the presence of severe ocular surface disease is a relative contraindication to penetrating keratoplasty. Occasionally, however, keratoplasty is necessary if the eye is to be preserved. Such eyes are demonstrably at greater risk as a result of the unhealthy ocular surface with dry eye, abnormal mucus secretion or even trichiasis. In all these situations every attempt should be made to improve the ocular environment with tear substitutes, punctal occlusion, mucolytics, lid margin hygiene and lid plastic surgery as appropriate.

Only one infection in this series was related to contact lens wear, a bandage lens used for a persistent epithelial defect. This apparently low incidence is perhaps surprising, but may reflect reluctance to use contact lenses after keratoplasty.

The results of this series suggest that mircobial keratitis following penetrating keratoplasty carries a poor prognosis both from a visual and tectonic point of view.

Only $23 \%$ of cases retained a clear graft, (which nonetheless was of benefit in patients in whom there were risk factors which were a potential threat to the other eye), the graft decompensated in $13 \%$ of cases and perforated in $17 \%$. Overall $53 \%$ of cases were regrafted.

In conclusion, several factors have been confirmed which increase susceptibility to microbial keratitis after penetrating keratoplasty. Loose sutures, epithelial defects and recurrent herpetic disease may compromise the epithelial barrier to infection. Long term use of antibiotics and steroids may both affect the conjunctival bacterial flora and alter host defence mechanisms. Diagnosis and treatment may be delayed because of patient's poor visual acuity and diminished corneal sensation. This complication of penetrating keratoplasty although not common carries high ocular morbidity and therefore demands. early recognition and aggressive treatment.

\section{References}

${ }^{1}$ Tuberville AW and Wood TO: Corneal ulcers in corneal transplants. Curr Eye Res 1981, 1: 479-4.

${ }^{2}$ Lamensdorf M, Wilson LA, Waring GO, Cavanagh HD: Microbial keratitis after penetrating keratoplasty. Ophthalmology 1982, 89 (Suppl), 124.

${ }^{3}$ Morris RJ and Bates AK: Changing indications for penetrating keratoplasty. Eye 1989, 3: 455-9.

${ }^{4}$ Dart JKG: Bacterial keratitis in contact users. $\mathrm{Br}$ Med J 1987, 295: 959-60.

${ }^{5}$ Fong LP, Ormerod LD, Kenyon KR, Foster CS: Microbial keratitis complicating penetrating keratoplasty. Ophthalmology 1988, 95: 1269-75.

${ }^{6}$ Harris DJ, Stulting RD, Waring GO, Wilson LA: Late bacterial and fungal keratitis after corneal transplantation. Ophthalmology 1988, 95: 1450-7.

${ }^{7}$ Al-Hazzaa SAF and Tabbara KF: Bacterial keratitis after penetrating keratoplasty. Ophthalmology 1988, 95: 1504-8.

${ }^{8}$ Meisler DM, Langston RHS, Naab TJ, Aaby AA, McMahon JT, Tubbs RR: Infectious crystalline keratopathy. Am J Ophthalmol 1984, 97: 337-43.

${ }^{9}$ James CB, McDonnell PJ, Falcon MG: Infectious crystalline keratopathy. $\mathrm{Br} J$ Ophthalmol 1988, 72: 628-30.

${ }^{10}$ Driebe WT and Stern GA: Microbial keratitis fol. lowing corneal transplantation. Cornea 1983, 2: 41-5. 